\title{
EFFECT ON LINKAGE MAPS OF SELECTION OF CROSS- OVERS BETWEEN CLOSELY LINKED MARKERS
}

\author{
E. CALEF \\ Department of Genetics, University of Glasgow
}

Received 5.x.56

SeVERAL cases of anomalous frequencies of multiple exchange in small chromosome segments have been reported in the literature. Examples are Drosophila virilis (Demerec, 1926), Neurospora (Giles, I95I ; M. Mitchell, I955 : St Laurence, I956), Drosophila melanogaster (Sturtevant, I952), maize (Laughnan, I952), E. coli (Rothfels, I952), Saccharomyces (Lindegren, I953), and Aspergillus (Pritchard, I955). These cases have one feature in common, that is, they show an excess of double exchanges: therefore they have a direct bearing on two of the most important problems of genetics, the linear arrangement of the genetic material and the mechanics of exchange.

Selection for wild-type crossovers from trans heterozygotes for two adenineless alleles in Aspergillus nidulans was found to be associated with a lengthening of the map in adjacent segments of the chromosome (Pritchard, 1955). The increase was up to about roo times over the length based on mapping by the analysis of random strands. This same situation was found in another group of adenineless mutantsthe object of the present paper-which turned out to be particularly suitable material for the problem under investigation; namely, the mechanism of crossing over in very short chromosome regions.

\section{EXPERIMENTAL}

(i) Materials

The techniques and the symbols are those described by Pontecorvo (1953). The four adenineless mutants used in these experiments, $\operatorname{adg}\left(\mathrm{S}_{5} \mathrm{D}_{1}\right), \operatorname{ad} \mathrm{I}_{3}\left(\mathrm{~S}_{5} \mathrm{C}_{4}\right), \operatorname{ad} \mathrm{I}_{5}\left(\mathrm{~S}_{5} \mathrm{E}_{4}\right)$ and $\operatorname{ad} \mathrm{I}_{7}\left(\mathrm{~S}_{5} \mathrm{E} 6\right)$ were obtained by Macdonald and Pontecorvo (1953) by U.V. treatment of a biotinrequiring strain followed by "starvation". They require adenine hydrochloride at an optimal concentration of $0.09 \mathrm{mg}$. per $\mathrm{ml}$. of medium; adenosine and hypoxanthine would also support their growth. The four strains are indistinguishable by means of growth response tests. All are self-sterile as most other adenineless mutants of $A$. nidulans. All crosses two by two are also sterile, except the cross $\operatorname{ad} \mathrm{I}_{5} \times a d_{\mathrm{I}} 7$ which gives only crossed perithecia and no selfed. Only these two mutants were extensively used in crosses. Both germinate on media lacking adenine but do not grow any further. The back mutation rate, which was measured in conidia, was very low: only one supposed reversion of $a d \mathrm{I}_{5}$ was found in about $1 \mathrm{O}^{9}$ conidia tested of each mutant. Self-sterility, cross-fertility, low back 
mutation rate and nutritional behaviour made these two mutants (ad I 5, ad I 7) particularly suitable for the present work.

TABLE I

Localisation of $\operatorname{ad} 15$ and $\operatorname{ad} 17$ by non-selective analysis

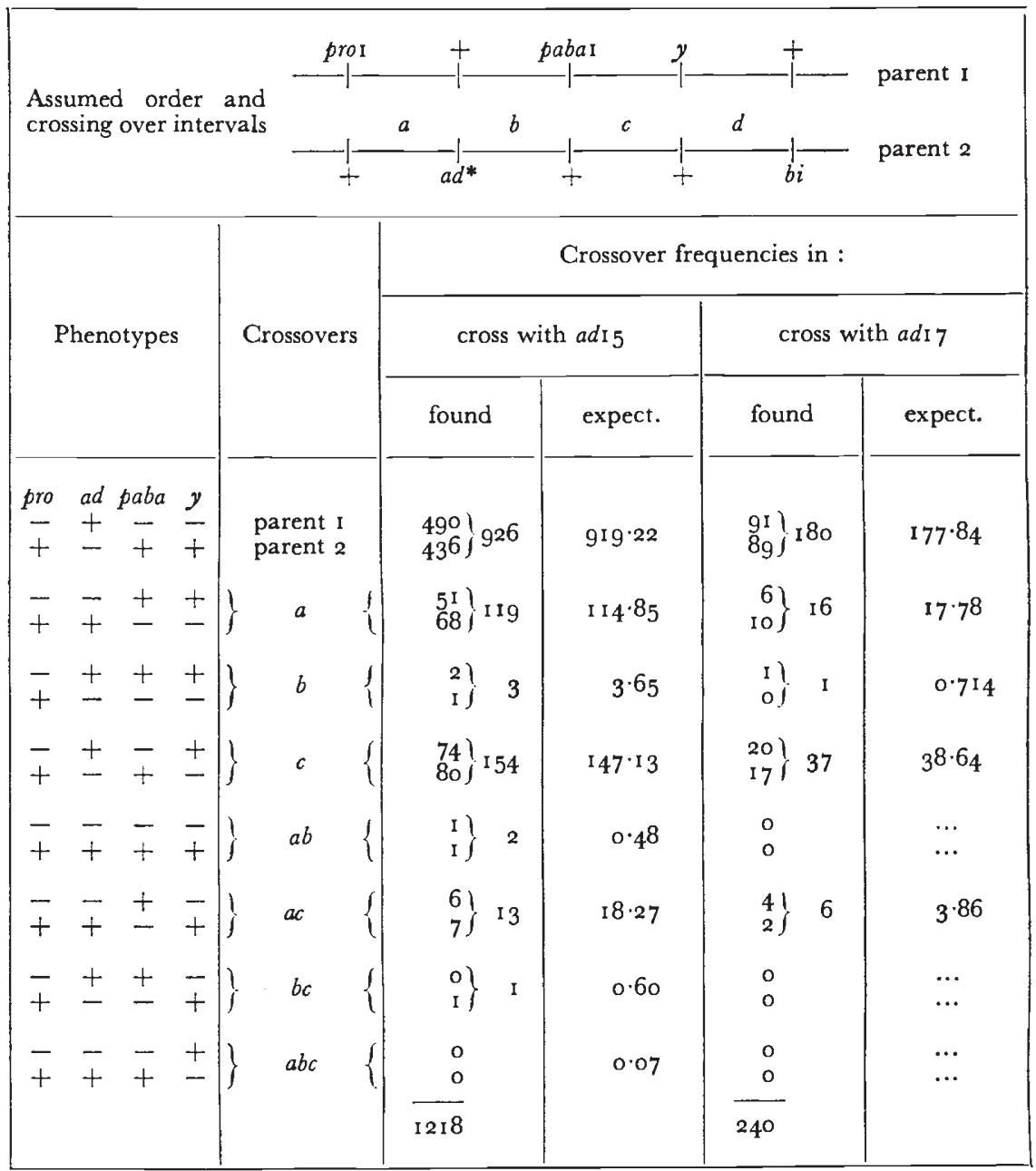

* Stands for $a d 15$ or $\operatorname{ad} \mathrm{I} 7$ according to the cross.

\section{(ii) Experiments}

The mutants $a d_{\mathrm{I}} 5$ and $a d_{\mathrm{I}} 7$ were known to be related to $a d 9$ which is very closely linked to pabaI ; heterokaryons $a d 9+a d \mathrm{I}_{5}$ and $a d 9+a d_{\mathrm{I}} 7$ were adenine requirers, suggesting allelism (Pritchard, unpublished).

Equally, the heterokaryon adg $+a d \mathrm{I} 3$ turned out to be adeninerequirer. These crude tests suggested that all four mutants might belong to one cluster of alleles. 
Starting from this information $a d_{1} 5$ and $a d_{1} 7$ were localised by crossing the original strains $a d \mathrm{I}_{5} b i$ and $a d \mathrm{I}_{7} b i$ with the strain proı paba 1 $y$. A random sample of crossed spores was first examined

TABLE 2

Localisation of $\operatorname{ad} \mathrm{I} 5$ and $\operatorname{adI} 7$ by selective analysis (selection for $\mathrm{ad}^{+} \mathrm{pabaI}^{+}$)

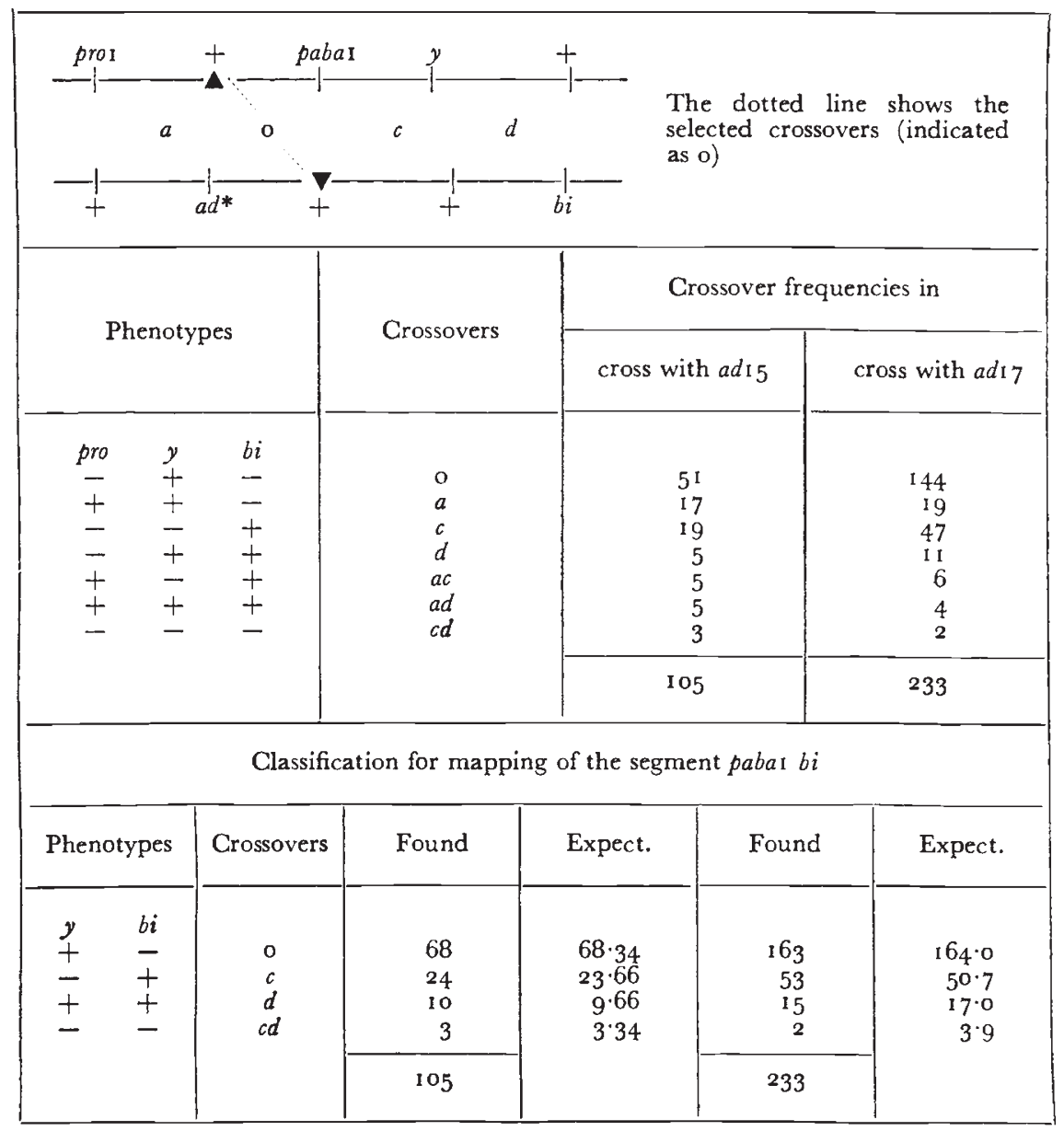

* Stands for $a d 15$ or $a d 17$ according to the cross.

$\Delta \boldsymbol{V}$ Indicates the points between which crossing over is selected.

with the results shown in table $\mathrm{I}$. In cross $a d \mathrm{I} 5 b i \times p r o$ I pabar $y$ out of 1218 ascospores examined there were 6 recombinants in the interval $a d-p a b a$. Three of them represented also a further exchange either in region $a$ or region $c$. In cross $a d \mathrm{I}\rangle$ bi $\times$ pro paba $y$ only one crossover in $b$ was found out of 240 ascospores. These data gave a first indication of the location of the mutants : both $a d \mathrm{r} 5$ and $a d \mathrm{r} 7$ are closely linked to paba and most likely between pro and paba. 
Decisive evidence came from selecting recombinants $a d^{+} p a b a \mathrm{I}^{+}$ (independent of both adenine and $p$-aminobenzoic acid) from the same pools of ascospores used in the non-selective platings of table $\mathrm{I}$. The data are reported in table 2. Unmistakably the sequence is :

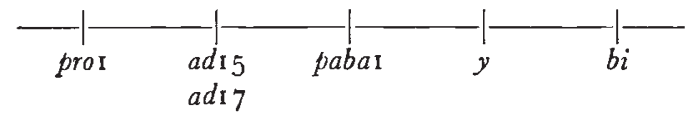

TABLE 3

Estimation of the recombination fraction between pabar and $\operatorname{adr} 5$ or $\operatorname{adr} 7$

\begin{tabular}{|c|c|c|c|}
\hline \multicolumn{2}{|c|}{ Colonies on complete medium } & \multicolumn{2}{|c|}{$\begin{array}{l}\text { Colonies on minimal medium } \\
\text { supplemented with proline and } \\
\text { biotin }\end{array}$} \\
\hline \multicolumn{2}{|c|}{ Cross involving } & \multicolumn{2}{|c|}{ Cross involving } \\
\hline $\operatorname{ad}_{\mathrm{I}} 5$ & $\operatorname{ad}_{1} 7$ & $\operatorname{adi}_{15}$ & $\operatorname{ad}_{17}$ \\
\hline $\begin{array}{c}\text { No./plate } \\
4 \text { I } \\
34 \\
44 \\
2 \text { I } \\
33 \\
28 \\
34 \\
3 \text { I } \\
34 \\
\text { I } 7\end{array}$ & $\begin{array}{c}\text { No./plate } \\
46 \\
48 \\
65 \\
46 \\
43 \\
45 \\
66 \\
64 \\
4^{2} \\
64\end{array}$ & $\begin{array}{c}\text { No./plate } \\
8 \\
8 \\
9 \\
6 \\
\text { 10 } \\
7 \\
7 \\
\text { 10 } \\
4 \\
3\end{array}$ & $\begin{array}{c}\text { No./plate } \\
\text { I I } \\
4 \\
9 \\
\text { I } 7 \\
\text { I } 1 \\
5 \\
6 \\
\text { I } 2 \\
3 \\
7\end{array}$ \\
\hline 317 & 529 & $7^{2}$ & 85 \\
\hline
\end{tabular}

Platings of ascospores from a single perithecium of crosses :

$\operatorname{ad} \mathrm{I} 7$ (or $a d_{\mathrm{I}} 5$ ) bi $\times$ pror pabai $y$

The same suspension of ascospores from each cross was plated undiluted on selective medium, diluted I/6o (a separate dilution for each plate) on complete medium. The double of the ratio of mean number of colonies on selective medium to that on complete medium $(\times 60)$ is an estimate of the recombination fraction between $a d_{1} 5$ (or $a d \mathrm{I}_{7}$ ) and pabar.

Using new pools of ascospores from crossed perithecia of the same crosses, the best estimate of the recombination value between ad and $p a b a$ was calculated from the ratio of recombinants requiring neither adenine nor $p$-aminobenzoic acid to the total of viable ascospores. This ratio was obtained from plating two parts of a single suspension : plating ( $\mathrm{r}$ ) on selective medium (devoid of $p$-aminobcnzoic acid and adenine), ten separate samples; plating (2) on complete medium, ten separately diluted samples. The dilution in plating (2) was I $/ 60$ (table 3 ). The recombination values calculated from these data are 
$3.3 \times \mathrm{IO}^{-3}$ for the interval $a d \mathrm{I}_{5}-p a b a \mathrm{I}$ and $2.4 \times \mathrm{ro}^{-3}$ for the interval $a d \mathrm{I} 7-p a b a \mathrm{I}$. The difference between these values is not significant.

Similar crosses involving $a d 9$ and $a d \mathrm{I}_{3} 3$ located also these two mutants at practically the same position as $a d_{I} 5$ and $a d_{1} 7$. Full details are not given here because they are irrelevant for the present paper.

Recombination between adi5 and adi 7 . The experimental evidence so far was still compatible with the possibility that adi 5 and $\operatorname{ad} \mathrm{I} 7$ might represent repeated isolation of the same mutant. However, as mentioned before, crosses between $a d_{1} 5$ and $a d I_{7}$ were fertile: showing that the two mutants were complementary (in respect of fertility), i.e. not identical at least functionally. As an extrapolation, it was expected that adI 5 and adi 7 represent mutations at two different sites (Pontecorvo, I952), distinguishable by means of crossing over. To test this expectation, two groups of crosses were made, differing from one another in that the two adenine mutants were in reciprocal positions in respect of the other markers used. From the two groups, only the following two crosses will be reported in full detail here because though all of them gave concordant results only these two were planned for a comparison. These crosses are :

Cross A

\begin{tabular}{|c|c|c|c|c|c|c|c|}
\hline & & $a$ & & $c$ & & & \\
\hline Parent I & + & $a d 1_{7}$ & + & paba1 & $y$ & + & $w_{n}$ \\
\hline ent 2 & pro I & + & $\operatorname{ad} 15$ & + & + & $b i$ & + \\
\hline
\end{tabular}

Cross B

\begin{tabular}{|c|c|c|c|c|c|c|c|}
\hline & & & $b$ & $c$ & & & \\
\hline Parent $I$ & + & + & $a d I_{5}$ & paba $\mathrm{I}$ & $y$ & + & $w_{n}$ \\
\hline Parent 2 & fror I & $\operatorname{ad} x_{7}$ & + & + & + & $b i$ & + \\
\hline
\end{tabular}

Large numbers of ascospores could be readily collected from these crosses. That all these spores were of crossed origin was shown by testing about 50 perithecia from each of cross A and B. These perithecia were picked at random and separately streaked on complete medium; they invariably gave rise to three-colour streaks (green, white and yellow). Green and white are parental colours; yellow is recombinant (Hemmons, Pontecorvo and Bufton, 1953). Both from crosses A and B large numbers of spores were collected free from conidial contamination. Samples of these spores were plated on minimal medium supplemented with proline, $p$-aminobenzoic acid and biotin but not with adenine, and on complete medium. On complete medium the viable counts were roo per cent. in almost every case. On the medium without adenine only adenine-independent colonies grew, together with a few poorly growing heterokaryons and a few diploids. Heterokaryons and diploids are casily recognised (Pritchard, 1954) and have been removed from the analysis.

The frequency of real adenine-independent colonies was about $3 \times 10^{-6}$. 
The results of the selective platings are shown in table 4. The majority ( 57 per cent. in cross A, 59.5 per cent. in cross B) of the

\section{TABLE 4}

Segregation frequencies in crosses $A$ and $B$ among selected adenine-independent recombinants

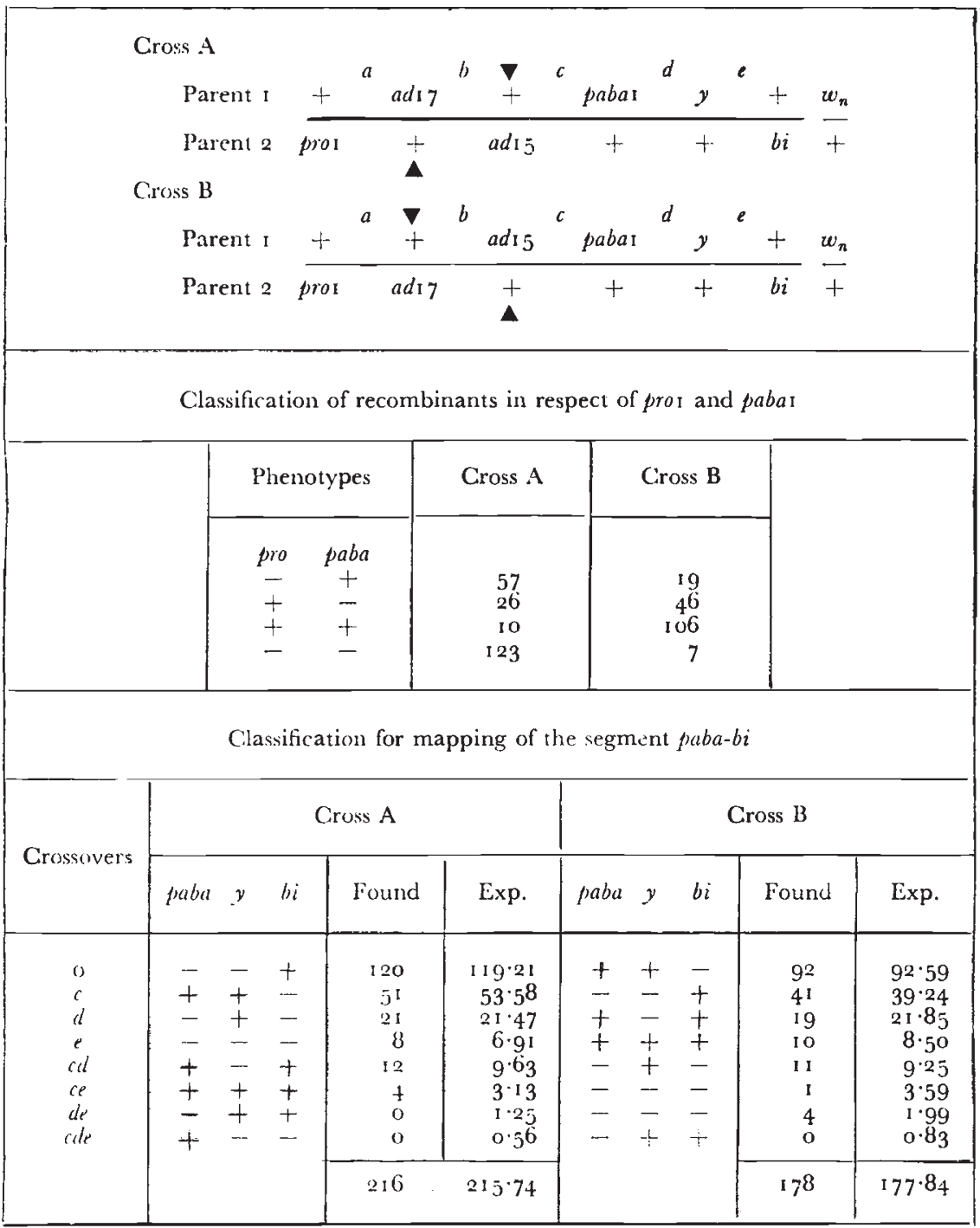

$\Delta \boldsymbol{V}$ Indicates the points between which crossing over is selected.

adenine-independent colonies are recombinant between pror and paba $\mathrm{I}$; in both crosses $\mathrm{A}$ and $\mathrm{B}$ the spectrum is in accord with the hypothesis of two sites of mutation and the sequence is the following :

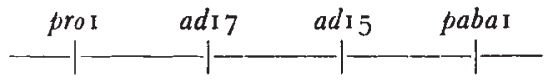


Keeping in mind that the two spectra are reciprocal, the results of the selective platings from the two crosses are homogeneous, i.e. viability effects could easily account for the minor differences observed which incidentally are not statistically significant. By pooling the data the following map is obtained :

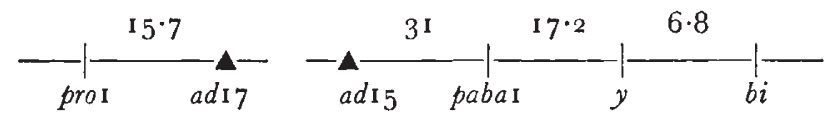

TABLE 5

Segregation frequencies in crosses $A$ and $B$ among non-selected ascospores

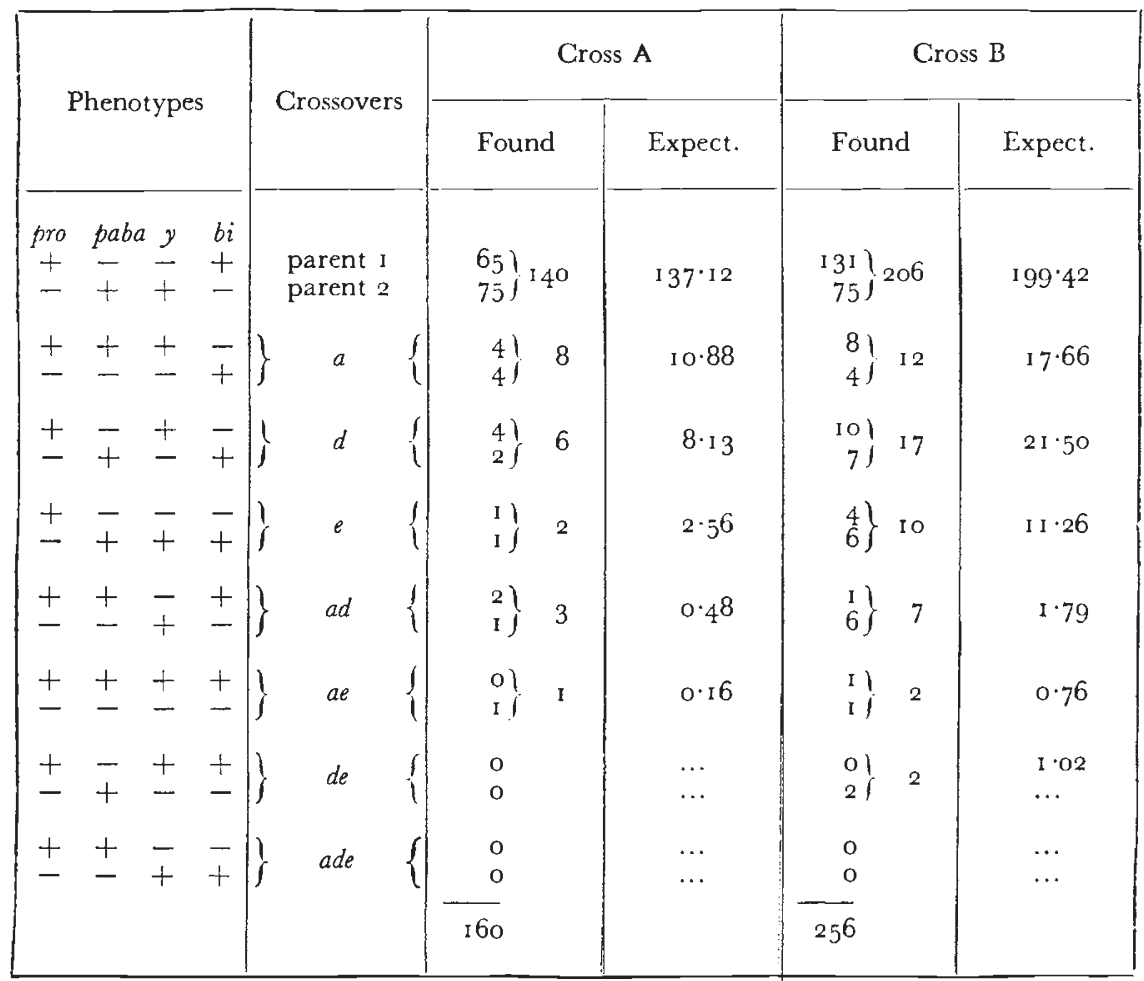

One-half of the recombinants in crosses A and B carried $w_{n}$ (white) epistatic to $y$ (yellow) and $y+$ (green). One-third of the whites were outcrossed to ascertain the presence of $y$ or $y^{+}$and estimate it for the rest.

This map will have to be compared with one based on the results of non-selective platings from the same crosses. The data are in table 5 .

In cross $\mathrm{B}$ the frequencies of the complementary crossover classes are heterogeneous (see for instance the parental classes, one of which is almost double the other). This heterogeneity is probably due to lower viability of prolineless spores which is known to occur on some batches of complete medium. (The possibility of contamination by selfed parental spores or by conidia has been ruled out.) Low viability of pro I would affect the formal analysis by increasing the crossover values of $a$ and $e$ and decreasing the coincidence value. 
In the relatively small samples of spores analysed in non-selective platings no recombination between $a d_{1} 5$ and $a d_{1} 7$ is expected, nor was it observed : the crosses behave as if homozygous $a d \mathrm{I} 5$ or $a d \mathrm{I} 7$ and can not yield information as to recombination in region $c$ (the segment of chromosome between $a d d_{1} 5$ and $\left.p a b a \mathrm{I}\right)$. However, these

TABLE 6

Comparison between maps based on selective analysis and those based on non-selective analysis in crosses heterozygous $\operatorname{adr} 5 / \operatorname{adr} 7$

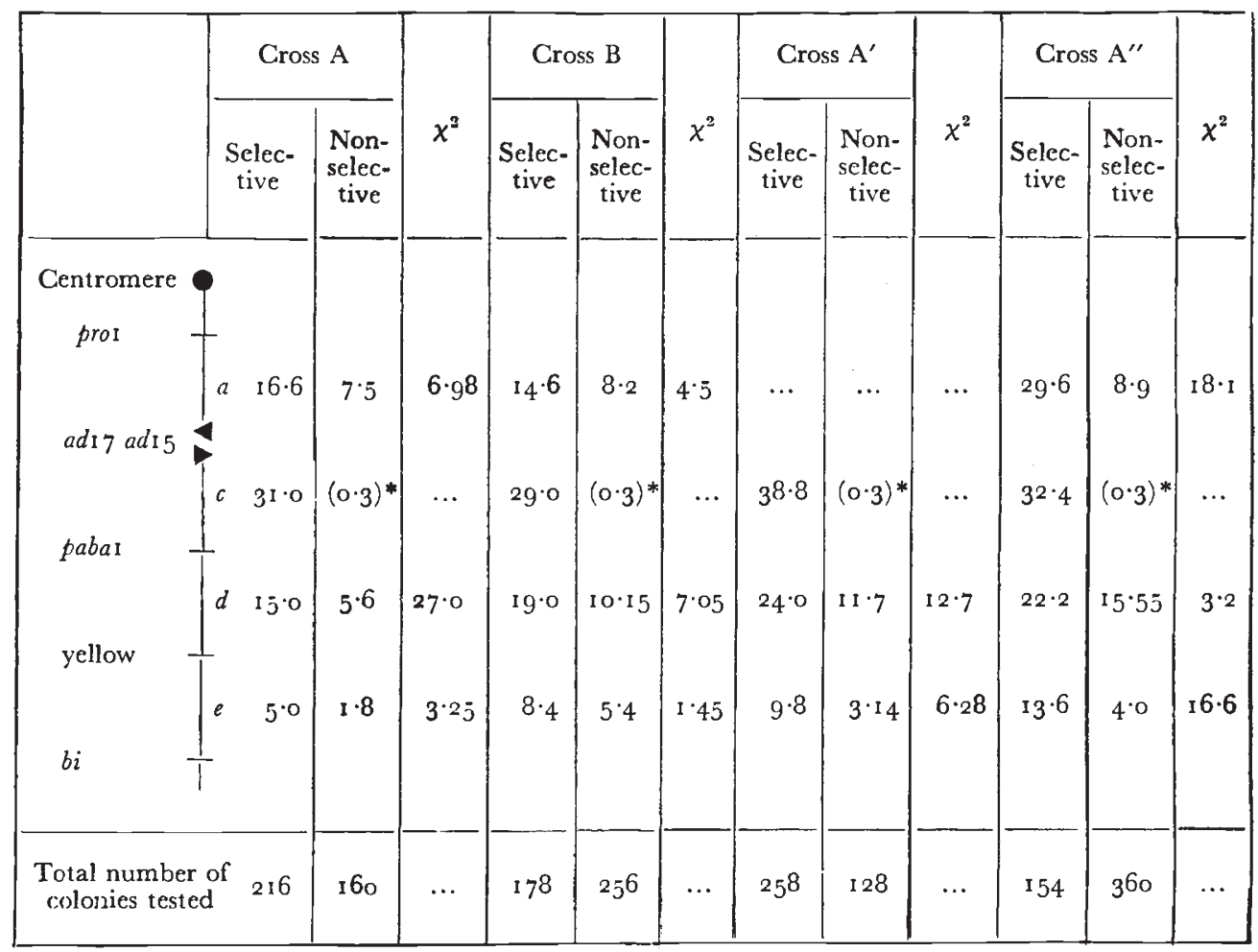

$\Delta \nabla$ Indicates the points between which crossing over is selected.

* The estimate in brackets derives from the experiment reported in table 3.

\begin{tabular}{ccccccc} 
Cross $\mathrm{A}^{\prime}:$ & + & $a d_{17}$ & + & $p a b a \mathrm{I}$ & $y$ & + \\
\cline { 2 - 6 } & + & + & $a d_{15}$ & + & + & $b i$ \\
Cross $\mathrm{A}^{\prime \prime}:$ & + & $a d_{\mathrm{I}} 7$ & + & $p a b a \mathrm{I}$ & $y$ & + \\
\hline proI & + & $a d_{15}$ & + & + & $b i$
\end{tabular}

data are valuable because they allow a comparison between selective and non-selective analysis within each cross. This comparison is shown in table 6 , on which the results of other similar crosses $\left(A^{\prime}\right.$ and $\mathrm{A}^{\prime \prime}$ ) are also included. 
The selective analysis (based on data involving a selected crossover between the two ad) gives a map-length for region $c$ some 30 units greater than that found as the average value in non-selective mapping (see table I). Again, for region $a$ selection gives a map-length some 7 units greater than that found without selection on the same sample of ascospores. The two segments $a$ and $c$ are both adjacent to the point at which the crossover leading to adenine independence was

TABLE 7

Comparison between maps based on selective analysis and those based on non-selective analysis in crosses pror pabai $\mathrm{y} \times \mathrm{adr} 5 \mathrm{bi}$ and pror pabai $\mathrm{y} \times \mathrm{adr} 7 \mathrm{bi}$ (from the data of tables $I$ and 2)

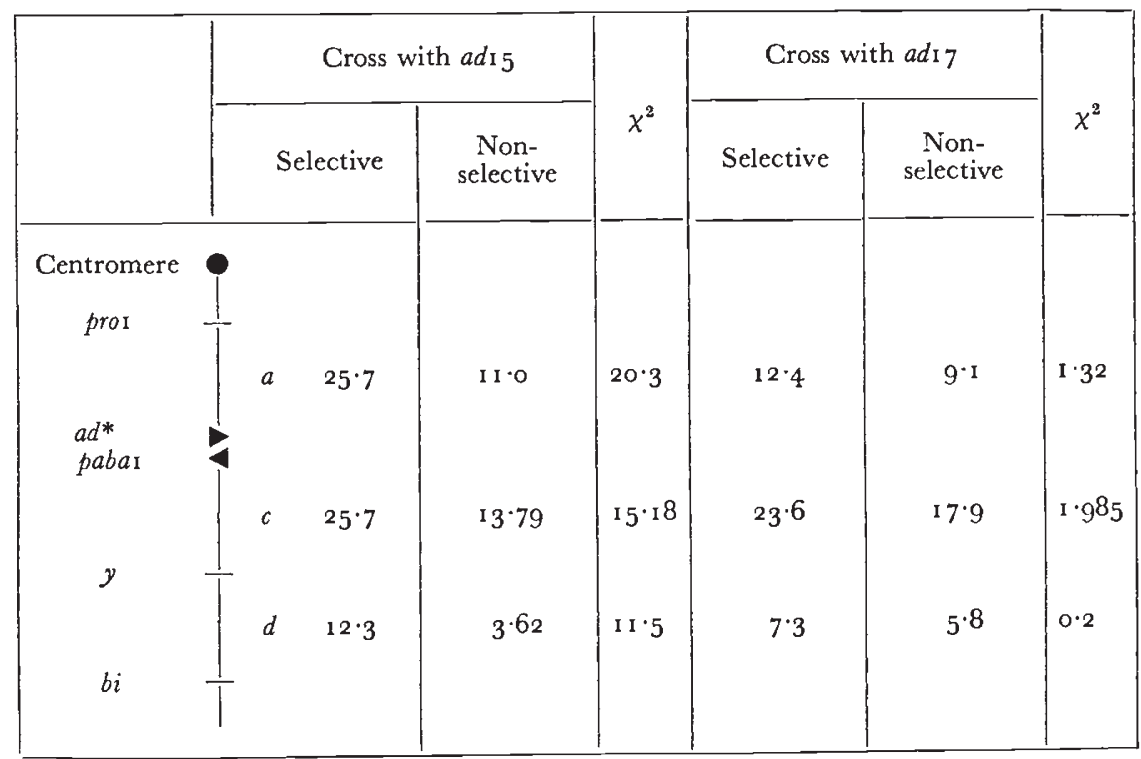

$\Delta \boldsymbol{V}$ Indicates the points between which crossing over is selected.

Stands for $a d_{1} 5$ or $a d_{1} 7$ according to the cross.

selected. Segments $d$ and $e$ which are farther away from the point of selection also show an increase in map-length though to a slighter extent.

That the increase in map-length is not a peculiar feature of selection of crossovers between $a d_{1} 5$ and $a d_{1} 7$, is shown by two further types of evidence. First, the data in tables $\mathrm{I}$ and 2, compared in table 7 , show that selection for $a d^{+} p a b a^{+}$crossovers, i.e. between two genes functionally quite distinct and less closely linked (recombination fraction of $\left.3 \times 10^{-3}\right)$, determines a small but significant increase of the map-length in both adjacent intervals.

Second, another experiment involving two complementary and closely linked prolineless mutants, proi and pro3 (Forbes, 1956) gave the same sort of results. The experimental procedure was the same as that in crosses $\mathrm{A}$ or $\mathrm{B}$, and the results are in table 8. 


\section{DISCUSSION}

(i) Origin of "prototrophic" colonies

Before discussing the experiments reported here it is necessary to be clear on what gives origin to the non-requiring colonies from

TABLE 8

Comparison between selective and non-selective mapping in cross

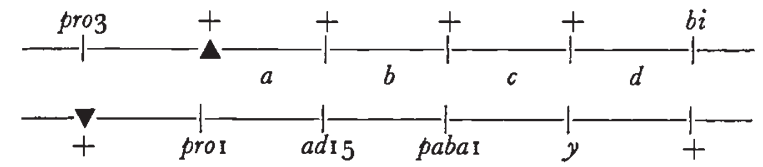

\begin{tabular}{|c|c|c|c|c|}
\hline \multirow{2}{*}{$\begin{array}{c}\text { Centromere } \\
\text { pro3 } \\
\text { pro } 1\end{array}$} & \multirow[b]{2}{*}{$a$} & Selective & Non-selective & $\chi^{2}$ \\
\hline & & $14 \cdot 18$ & $8 \cdot 6_{4}$ & 2.86 \\
\hline$a d_{1} 5$ & $b$ & 0.5 & 0.5 & $\ldots$ \\
\hline paba $\mathrm{I}$ & $c$ & $16 \cdot 2$ & 13.7 & 0.21 \\
\hline & $d$ & $4 \cdot 7$ & 4.07 & 0.09 \\
\hline \multicolumn{2}{|c|}{$\begin{array}{l}\text { Total number of colonies } \\
\text { tested }\end{array}$} & 191 & 196 & $\cdots$ \\
\hline
\end{tabular}

$\nabla \Delta$ Indicates the points between which crossing over is selected.

crosses $\operatorname{ad}_{\mathrm{I}} 7 \times a d_{\mathrm{I}} 5$ and $p r o 3 \times$ proI. Discussion of the mechanism leading to their formation is pertinent because the explanation based on crossing over between two sites of mutation might be incorrect since it implies an increased frequency of exchange near the selected locus.

This point has been discussed by Pritchard (1955) when he first found association of prototroph formation with increased recombination near the locus involved. His conclusions based on mitotic analysis - which favour crossing over-are very convincing.

In the present case the selection of adenine-independent recombinants in pairs of crosses with the reciprocal arrangements of markers (crosses $\mathrm{A}$ and $\mathrm{B}$ ) gives spectra of segregation with mirror image patterns (see data in table 4). Again crossing over seems to be the most economical explanation. 
Further evidence in favour of crossing over derives from Forbes' (1956) work on prolineless mutants, which Strickland (unpublished) was able to supplement by tetrad analysis. All the asci containing proline-independent recombinants also contained the complementary double mutant recombinants, besides the non-crossover strands.

\section{(ii) The lengthening of the maps in selective analysis}

Two groups of models have been put forward to explain cases of anomalous frequencies of what appear as products of multiple exchanges within small chromosome regions. First, those which suggest special mechanical devices of recombination either based on crossing over or other unknown mechanisms. Second, those which suggest formal kinds of explanation in which a coincidence value higher than one would result because of the inclusion in the calculation of meiotic products in which crossing over was absent.

In the former group one may put Winkler's (1930) "gene conversion ", the model of copying choice of Lederberg (1954), and the explanation suggested by Pritchard (1955). The latter group includes those by Rothfels (1952), Sturtevant (1954), Cavalli and Jinks (1954 and 1956). While the first group might have an advantage in the possibility of providing also a model for the duplicative process of the chromosome, the second is more in keeping with the well-established production of complementary strands in crossing over. These two categories are not necessarily conflicting; in fact the final explanation valid for all the data that are being collected might turn out to be an integrated form of both of them.

In A. nidulans Pritchard (1955) found a great increase, compared to the expectation based on mapping by random strand analysis, in the number of exchanges in a segment of chromosome next to one within which a crossover between adenineless alleles was selected. A further investigation by Forbes (1956) on two other groups of alleles (proI and pro3) gave the same pattern of recombination. The present investigation yields results strictly similar to those of Pritchard; it shows an increase in map length with the following features: (a) the effect spreads beyond the chromosome segments adjacent to the point of selection (table 6 compares maps from selective and from non-selective analysis and shows the increases in map-length in nonadjacent regions and the falling-off of this effect with the distance from the point of selection); (b) the magnitude of increase appears to be related to the closeness of linkage between the markers used for selection. Table 6 , where selection was for $a d_{1} 5^{+} a d \mathrm{I} 7^{+}$, shows the effect of a selection Iooo times stronger than table 7 , where selection was for $a d \mathrm{I}^{+} p a b a \mathrm{I}^{+}$, or table 8 , where selection was for $\operatorname{pro3}^{+}$proI $^{+}$.

A tentative model for a theory of incomplete pairing offers an interpretation of the experimental data. 
I assume that pairing, ${ }^{*}$ as a necessary condition for crossing over, is not complete. In any one population of cells in meiosis for any one chromosome section there is a fraction with no pairing. As a necessary first approximation it is assumed that the chromosome segments which are paired have a random distribution along the chromosome length.

The main consequences of the theory are that the increase in map-length due to selection is the higher the more stringent is the selection, and that this increase becomes smaller with distance from the point of selection.

Let $l$ be the length of the pairing segment, $x$ the length of the segment within which crossovers are selected, $a$ and $c$ the two chromosome segments on each side of $x$. The frequency of recombination in $c$ in all the zygotes in which a crossover in $x$ is selected will be :

$$
f_{c}=\frac{1}{2} \frac{l}{l+x} \text {. }
$$

(The factor $\frac{1}{2}$ is taken for a case of maximum frequency of recombination in $l$; it will be less than this if the incidence of crossing over in the paired segment is not very high.)

If $l$ (the length of the paired segment) were constant from cell to cell, though its position varied at random over the whole chromosome length, then (I) would give the expected relationship between $f_{c}$, an observable quantity, and $x$ a quantity that can be varied by choosing for selection markers varying in their closencss. The curve thus obtained will be of the form given in fig. I for $l=\mathrm{I}$, where $x$ represents a multiple of $l$. It will be seen that for the experiment in which selection is for crossovers between $a d \mathrm{I} 7$ and $a d \mathrm{I} 5$ - which are at a distance of $3 \times \mathrm{rO}^{-6}$ - the observed recombination $(0.30)$ in the segment $a d \mathrm{r} 5-p a b a \mathrm{I}$ corresponds to a value of $x=0 \cdot 78$, i.e. the length of the pairing segment would be $\mathrm{I} \cdot 4$ times the length of the segment in which the crossover was selected, i.e. 0.00038 map units.

If, on the other hand, $l$ were variable from cell to cell, even assuming an extreme case of a skew distribution of the form :

$$
d \phi(l)=k e^{-k l} d l
$$

the shape of the curve would not vary substantially (fig. I.), though its values would be somewhat lower. In this case the observed recombination in the segment adr 5-pabar would correspond to a value of $x=$ 0.35 , and the pairing segment would be about 0.001 map units.

The applicability of this theory rests on the symmetry of the increases on both sides of the point of selection. Let us, thus, consider the increase in segment $a$ (between pros and $a d_{1} 7$ ). The value of recombination in $a\left(p_{s}\right)$ after selection should be :

$$
p_{s}=p_{r}+p_{x}-p_{r} \times p_{x}
$$

* The term "pairing", as used here, does not assume any relation to cytologically visible pairing as observed in prophase of meiosis. The term is unfortunate but it was used first by Rothfels (1952) and later by Cavalli and Jinks (1954, 1956) with the same meaning as here. Pritchard (1955) used the less confusing expression " effective pairing". 
where $p_{r}$ is the recombination fraction in non-selective analysis and $p_{x}$ the expected increase. On the basis of the values in table 6 , the expected value works out to be $p_{s}=0.356$, while, in fact, the found mean value is less than $0 \cdot 16$.

This model was built on the assumption that the pairing segments are distributed at random. The data clearly do not fit this simple form of the model. The theory of incomplete pairing stands on the basis of the qualitative results but the two first approximations, randomness of location and of size of the paired segments, are untenable.

This model concerns only segments adjacent to the point of selection. A more complicated one would be necessary to evaluatc

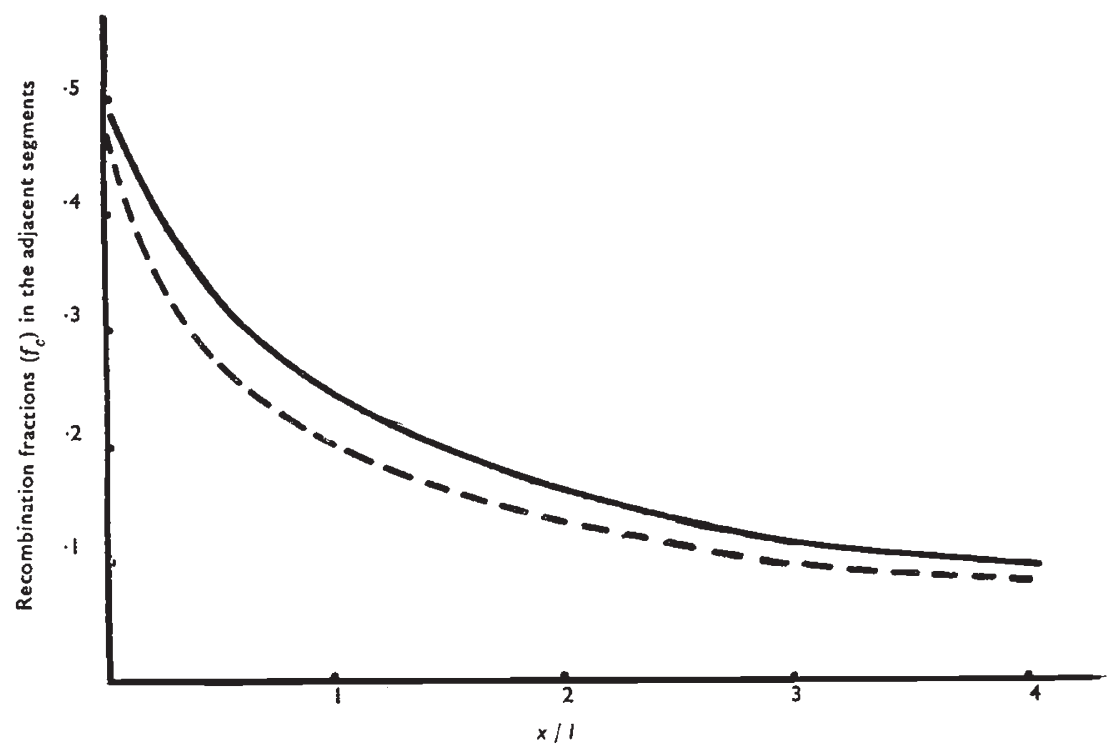

FIG. I-Relationship between recombination fractions in an adjacent segment and length $x$ of the segment where crossovers are selected. $x$ is cxpressed as a multiple of $l$ the length of the pairing segment. The two curves are for constant and highly variable length of the pairing segment, respectively.

the expected lengthening in non-adjacent segments. At the moment I will confine the discussion to its qualitative aspects.

The data, though limited, are sufficient to show that lengthening of non-adjacent segments occurs when the strength of selection is of the order required in selection of $a d \mathrm{I}_{1} 7^{+} a d_{\mathrm{I}} 5^{+}$(as in crosses $\mathrm{A}$ and $\mathrm{B}$ ). The problem now is whether this lengthening has the same cause as that in the adjacent segments. In the light of the theory of incomplete pairing, this would mean that at least some of the pairing segments are long enough to cover the portion of chromosome from the point of selection to the last but one marker, a length of 15 units on the non-selective map and 45 units on the selective ones.

As an alternative explanation, let us suppose that the increased recombination in non-adjacent segments were due to selection for 
products of cells in meiosis with different proneness to crossing over. Paraphrasing Sturtevant's example (1954, p. 238), suppose we were analysing a mixed population of gametes from males and females of Drosophila. The selective analysis would discard the male gametes, and all the map-lengths would be increased by a constant factor dependent on the proportion of non-crossover gametes, but independent of the distance from the selected point. The results reported in table 8 seem to exclude this alternative. Here, in fact, the segment adjacent to the point of selection is substantially increased, in selective analysis, while the three segments beyond it are not. The explanation in terms of the theory of incomplete pairing seems to be the simplest. Due to the relatively small strength of selection in this case, the longer paired segments are not selectively picked up as much as in experiments like those of selection for $a d \mathbf{I} 5^{+} a d_{1} 7^{+}$.

Finally there is evidence in favour of incomplete pairing which is complementary to that discussed so far. That is, coincidence values greater than $\mathrm{I}$ are found in non-selective analysis. This is the necessary counterpart, expected on theory, of the lengthening of the maps in selective analysis. None of the previous work on Aspergillus nidulans had detected any kind of interference because it dealt with regions too large to provide a sensitive test.

The evidence is as follows. The experiment on localisation of $a d_{\mathrm{I}} 5$ reported in table $\mathrm{I}$ includes two adjacent segments ( $a$ and $b$ ) of total length less than ten units. The coincidence value found there is about 4. In crosses $A$ and $B$ the non-selective analysis again gives coincidence values in excess of $I$ over total lengths of about I 3 units. Although only few recombinants are available for these estimations, the tendency is clear and highly suggestive.

\section{SUMMARY}

I. The formation of rare prototrophs among ascospores from crosses between two adenineless mutants $\left(a d_{1} 5\right.$ and $\left.a d_{1} 7\right)$ of Aspergillus nidulans is most probably due to the occurrence of crossing over between them. Reciprocal crosses yield strictly complementary segregation patterns.

2. Selection for crossovers between very closely linked markers lengthens the linkage maps, as compared with the maps based on non-selective analysis.

3. The increase in recombination frequencies has its maximum near the point of selection. It spreads over segments of chromosome beyond those adjacent to the point of selection. And it is the greater the closer the linkage between the markers used for selection.

4. Parallel to the increase of recombination frequencies in selective analysis, coincidence values higher than I are found in non-selective analysis between pairs of segments within an overall length of about Io units. 
5. These increases can be accounted for by assuming that pairing is not complete along the whole length of every bivalent in every cell in meiosis, but partial and variable in length and position. Selection for crossovers at one point would also select the products of those cells in meiosis in which the probability of pairing around the selected point is greater than average.

Acknowiledgments.-This work, which is part of a general programme supported by the Nuffield Foundation, was carried out under the tenure of a Nuffield Research Scholarship. The author is deeply indebted for advice and criticism to Professors G. Pontecorvo and L. L. Cavalli-Sforza.

\section{REFERENCES}

CAVAlli-SFORZA, L. L., AND JINKS, J. L. 1954. Observations on the genetic and mating system of $E$. coli $\mathrm{K}_{\mathrm{I} 2}$. Caryologia, 6 (suppl.), $967-969$.

CAVAlli-SfORZA, L. L., AND JiNks, J. L. 1956. Studies on genetic system of $E$. coli KI 2. 7. Genet., 54, 87-ז I 2 .

DEMEREC, M. 1926. Reddish; a frequently mutating character in $D$. viriiis. P.N.A.S., I2, I1-1 6 .

FORBES, E. C. 1956. Recombination in the pro region of Aspergillus nidulans. Microb. Genet. Bull., I3, 9-1 1.

GILES, N. H. I951. Studies on the mechanism of biochemical mutants of $\mathcal{N}$. crassa. Cold Spr. Harb. Sym. quant. Biol., I6, 283-313.

hemmons, L. M., PONTECorvo, G., and BUfton, A. W. J. 1953. The genetics of Aspergillus nidulans. Adv. Genet., 5, 194-201.

LAUGHNAN, J. R. 1952. The action of the allele form of the gene A in maize. IV. On the compound nature of $A^{b}$ and the occurrence and action of its $A^{d}$ derivatives. Genetics, 37, 373-395.

LederberG, J. 1954. Recombination mechanism in bacteria. 7. cell. comp. Physiol., 45 (suppl. 2), 75-107.

Lindegren, c. c. 1953. Gene conversion in Saccharomyces. 7. Genet., 5I, 625-637. macdonald, K. D., AND ponteconvo, G. 1953. The genetics of A. nidulans. Adv. Genet., 5, I 59 - I 70.

MITCHELL, M. M. 1955. Aberrant recombination of pyridoxin mutants of Neurospora. P.N.A.S., 4I, 215-220.

PONTECORVO, G. 1952. The genetical formulation of gene structure and action. Adv. Enzymol., 13, 121-149.

PONTECORvo, G. 1953. The genetics of $A$. nidulans. Adv. Genet., 5, 141-238.

PRITChARD, R. H. I954. Ascospores with diploid nuclei. Caryologia, 6 (suppl.), 1117.

PRITCHARD, R. H. I 955. The linear arrangement of a series of alleles of $A$. nidulans. Heredity, 9, 343-37 I.

ROPER, J. A., AND PRITCHARD, R. H. 1955. The recovery of complementary products of mitotic crossing over. Nature, I75, 639 .

ROTHFELS, к. H. 1952. Gene linearity and negative interference in crosses of Escherichia coli. Genetics, 37, 297-311.

St. LaUrence, patricia. 1956. The $q$ locus of Neurospora crassa. P.N.A.S., $4^{2}$, I 89-194.

STURTEVAnT, A. H. 1952. A map of the fourth chromosome in Drosophila melanogaster based on crossing over in triploid females. P.N.A.S., 37, 405-407.

STURTEVANT, A. H. 1954. Evaluation of recombination theory. J. cell. comp. Physiol., 45 (suppl. 2), 237-242.

WINKLeR, H. I930. Die conversion der gene. Jena : Fischer. 